\title{
Probiotics for Prevention of Clostridium difficile Infection
}

\author{
John P Mills ${ }^{a}$, Krishna Rao ${ }^{a}$, and Vincent B Young ${ }^{a, b,{ }^{*}}$ \\ aDivision of Infectious Diseases, Department of Internal Medicine, University of Michigan School \\ of Medicine
}

${ }^{b}$ Department of Microbiology and Immunology, University of Michigan School of Medicine

\begin{abstract}
Purpose of review-Probiotics may prevent $C$. difficile infection (CDI), a leading healthcareassociated infection in the United States. However, prior studies were limited by heterogeneity in products and patient populations. Recent clinical evidence and new approaches to probiotic development are reviewed.
\end{abstract}

Recent findings-Probiotic use may reduce incident CDI in high risk populations by as much as $50 \%$, though prior clinical trials have yielded conflicting results. Combining probiotics with prebiotics improves growth and engraftment in the host. Bacillus clausii and Lactobacillus reuteri secrete compounds that directly inhibit $C$. difficile. Organisms that produce secondary bile acids, such as Clostridium scindens, enhance $C$. difficile colonization resistance. Non-toxigenic $C$. difficile, which provides nutritional niche competition, may prevent CDI. Refinements to fecal microbiota transplantation (FMT) blur the line between probiotics and FMT. These include a quality-controlled stool product (RBX2660), purified Firmicutes spores (SER-109), and sterile fecal filtrate. Bacteriophages may treat CDI but have unknown safety and efficacy in humans.

Summary-There have been a number of advances in probiotics and our understanding of their role in prevention of CDI, but a number of important safety and efficacy questions remain. An improved understanding of the native microbiota structure and function will allow for continued development of rationally designed probiotic therapy to provide enhanced protection against CDI.

\section{Keywords}

Clostridium difficile infection; probiotics; prebiotics; fecal microbiota transplantation; bacteriophage therapy

\section{Introduction}

Clostridium difficile infection (CDI) is a scourge of the modern healthcare system, causing over 500,000 infections and 30,000 deaths, with annual costs totaling over \$1.5 billion. [1, 2] The Centers for Disease Control and Prevention currently lists CDI as an "urgent threat,"

\footnotetext{
*Corresponding author: Vincent B. Young, MD, PhD. 1150 W. Medical Center Drive, 1520B MSRB1, Ann Arbor, MI 48109. youngvi@umich.edu. Phone: 734-764-2237. Fax: 734-615-5534.

Conflicts of Interest

VBY has served as a consultant to Finch Therapeutics and Vedanta Biosciences.
} 
requiring a prioritization of public health resources to identify infection and limit transmission.[3]

An anaerobic, spore-forming, toxin-producing Gram-positive bacillus, $C$. difficile has been found to asymptomatically colonize $2-15 \%$ of patients, depending on underlying comorbidities and degree of prior healthcare exposure.[4, 5] C. difficile spores can persist in the environment and are resistant alcohol-based cleaning agents and quaternary ammonium compounds, resulting in high transmissibility.[6] The primary risk factor for development of $\mathrm{CDI}$ is exposure to antibiotics, which perturb the indigenous microbiota's structure and function, allowing for $C$. difficile acquisition and symptomatic disease.[7, 8] C. difficile acquisition can result in a wide range of outcomes from temporary asymptomatic colonization to fulminant pseudomembranous colitis. Current standard of care for CDI involves antibiotic treatment with metronidazole, vancomycin, or fidaxomicin.[9] Outcomes are suboptimal, with 12-64\% recurrence risk (median 22\%).[10] Thus, adjunctive therapies that can improve outcomes are actively being sought. In particular, given the important role that disturbances in the gut microbiota play in the pathogenesis of CDI, this microbial community represents a potentially novel therapeutic target.

Probiotics, a century-old concept, are defined as "live microorganisms which when administered in adequate amounts confer a health benefit on the host." $[11,12]$ In addition to the genus and species of a probiotic, the particular strain should be known, as health benefits can be strain-specific. Probiotics used to potentially prevent or treat CDI function through multiple possible mechanisms. [FIGURE 1] In many cases, these mirror postulated mechanisms by which the indigenous microbiota mediate colonization resistance against $C$. difficile.[7, 13]

Clinicians are often confused by conflicting data on probiotics for the prevention of CDI. This is compounded by the heterogeneity in prior clinical trials, utilizing different probiotic agents on varied patient populations and assessing different primary outcomes. Shen et al performed a recent meta-analysis of probiotic use for CDI prevention specifically in hospitalized adults receiving antibiotics.[14] They included 19 randomized controlled trials with 6261 patients treated with Saccharomyces boulardii, Lactobacillus spp,

Bifidobacterium spp, and Streptococcus spp, alone, or in combination. The relative risk (RR) of CDI in the probiotic treatment groups was 0.42 (95\% CI 0.3-0.57) without significant heterogeneity between studies $\left(I^{2} 0.0 \%, P=.56\right)$ and no evidence of publication bias. While rigorously conducted and focused on a specific high-risk population, the review was limited by significant variability in probiotic agents, case definitions, and placebo rates of AAD and CDI in the included studies. As such, significant questions remain: 1) what is the optimal probiotic strain or combination of strains, and 2) are they safe for use in immunocompromised hosts, who bear an outsized burden of CDI? Furthermore, current national guidelines for the treatment of CDI do not recommend routine probiotic use.[15, 16] As probiotic use in the context of CDI remains controversial, a summary highlighting active research in the field is warranted.

We take a mechanistic approach here to review the latest developments in probiotic research, focusing on their role in the prevention of primary and recurrent CDI. Methods to 
manipulate the indigenous microbiota span the spectrum from traditional single agent probiotics to FMT. We discuss products that include undefined microbial consortia, phage viruses, and bacterial-derived small molecule therapy, that fall outside the traditional probiotic definition. While we do discuss new FMT developments, we do not review FMT in depth, as has previously been done $[17,18]$.

\section{Trials of traditional single and combination agents}

In the past year there has only been a single large-scale clinical trial involving traditional single-agent probiotics. This was a multicenter, double-blind, randomized controlled trial assessing effectiveness of Saccharomyces boulardii for the primary prevention of antibioticassociated diarrhea (AAD) in hospitalized patients receiving antibiotics.[19]

This study of 477 patients, in which $C$. difficile infection was assessed as a secondary endpoint, failed to find a benefit of $S$. boulardii in the prevention of AAD or CDI. However, the study was underpowered to detect a difference due to under-enrollment and fewer than anticipated primary events.

A recent phase 2 study was the first to assess the ability of a probiotic to reduce duration of diarrhea for the initial episode of mild to moderate CDI. Use of a daily multi-strain capsule (Lactobacillus acidophilus NCFM, ATCC 700396; Lactobacillus paracasei Lpc-37, ATCC SD5275; Bifidobacterium lactis Bi-07, ATCC SC5220; B. lactis B1-04, ATCC SD5219) versus placebo for four weeks resulted in shorter duration of diarrhea ( 1 vs 0 days; $P=.039$ ). This exploratory pilot study of 33 patients will need to be replicated in a larger population to establish efficacy of probiotics as an adjunctive therapy for initial occurrence of CDI.[20]

\section{Synbiotics}

Prebiotics are non-digestible polysaccharides and oligosaccharides that promote the growth of specific genera of beneficial microorganisms by acting as a substrate for fermentation.[21, 22] When a prebiotic is administered with a specific probiotic to enhance the engraftment and growth of that microbe, the combination is termed a "synbiotic." A recent study assessed the ability of Lactobacillus plantarum Inducia ${ }^{\circledR}$ and the prebiotic xylitol to inhibit the germination of $C$. difficile spores.[23] Pre-incubation with $L$. plantarum and xylitol fully inhibited in vitro germination of $C$. difficile spores. CDI was attenuated in mice fed xylitol and $L$. plantarum 5-6 days prior to ampicillin and $C$. difficile administration, with a reduction in mortality from $44 \%$ to $22 \%$. No reduction in mortality occurred in mice who began synbiotic treatment after the $C$. difficile challenge. The generalizability of the findings is limited as it is a small in vitro and animal study. However, it does support further study on the enhancement of beneficial probiotic effects by dietary substrates and the relative temporal interaction of probiotic and antibiotic administration.

Another study assessed the ability of four different Bifidobacterium strains to inhibit in vitro $C$. difficile growth when co-cultured with various prebiotics.[24] Reduction in toxicity was observed when $B$. longum and $B$. breve were cultured in a cell line exposed to $C$. difficile cell-free supernatant using oligo-fructosaccharides as a carbon source. No beneficial effects were noted with use of inulin. Results suggest the optimal prebiotic substrate is strain specific. Further studies are needed to determine the optimal prebiotic substrate for each 
probiotic strain and to measure effects of prebiotic administration on the larger intestinal microbiota.

\section{Bacterial secreted compounds that inhibit the activity of $\boldsymbol{C}$. difficile toxins}

A study by Ripert et al investigated the in vitro ability of Bacillus clausii $\mathrm{O} / \mathrm{C}$ to neutralize $C$. difficile toxin, the major virulence factor of the pathogen.[25] Incubation of toxincontaining culture supernatants of $C$. difficile with supernatant from $B$. clausii protected mammalian cell lines from cytotoxic effects.[26] This was due to the production of a serine protease, M-protease, by B. clausii. Purified M-protease was able to protect Vero cells from the cytotoxic effects of $C$. difficile culture supernatants. Widespread screening of potential probiotic agents for enzymatic activities that can destroy $C$. difficile toxins may identify additional strains that can be combined for maximal inhibitory capacity.

\section{Direct inhibition of $C$. difficile}

Certain bacteria produce antibacterial compounds, that could prevent or treat CDI. This can include molecules such as the bacteriocin produced by Bacillus thuringiensis DPC 6431. Other bacteria produce non-protein antimicrobial compounds. Lactobacillus reuteri ferments glycerol to produce reuterin, an antibacterial substance with activity against numerous enteric pathogens, including $C$. difficile. A recent study by Spinler and colleagues utilized $L$. reuteri strain 17938, which displays high-level resistance to vancomycin, metronidazole, and fidaxomicin, making it an attractive option in patients receiving concomitant anti-CDI therapy.[27] These investigators utilized a mini-bioreactor system that contained a humanderived microbial community to compare the ability of L. reuteri 17938 with or without the addition of glycerol to inhibit $C$. difficile growth in bioreactors pretreated with clindamycin. The combination of $L$. reuteri and glycerol resulted in a 5-log reduction in the growth of $C$. difficile in these bioreactors $(P=.008)$.

Other investigators have examined the synergistic effects of microbe-derived antimicrobials against $C$. difficile. Durancin 61, a bacteriocin produced by Enterococcus durans, was purified and examined for in vitro activity against $C$. difficile strain ATCC 630 alone and in combination with the antibacterial compounds nisin and reuterin.[28] The combination of durancin 61 and reuterin exhibited the most potent inhibition and synergy. These results suggest that combinations of probiotic organisms may exhibit beneficial synergy. Given the fact that the normal microbiota is a complex community it is not surprising that the use of multiple probiotics can lead to synergy. As yet, we do not have information on which combinations of organisms may have the most beneficial effect.

\section{Restoration of bile acid homeostasis}

Bile acids play a key role in the physiology of $C$. difficile, with specific bile acids (generally primary, conjugated bile acids) serving as germinants for $C$. difficile spores and others having inhibitory activity on vegetative $C$. difficile.[29] Antibiotic treatment alters intestinal bile acid abundance and composition. Repopulation with $7 a$-dehydroxylating bacteria that convert primary to secondary bile acids could therefore provide improved $C$. difficile colonization resistance. 
A study by Pamer et al assessed changes in diversity and microbial composition by $16 \mathrm{~S}$ rRNA sequencing in mice treated with various antibiotics.[30] Variance in the microbial structure was used to identify specific taxa that provided resistance against CDI; organisms belonging to Clostridium cluster XIVa, most notably Clostridium scindens, a known producer of secondary bile acids, provided the greatest protection. A consortium of four bacterial species including $C$. scindens displayed attenuated CDI in antibiotic-treated, $C$. difficile-challenged mice. Sequencing data showed engraftment of $C$. scindens that was dose-dependently associated with protection from CDI, along with presence of $7 a-$ dehydroxylase capability in treated mice. These data suggest bacteria that synthesize secondary bile acids may form a crucial component of an engineered microbiota with resistance to CDI. This could potentially be accomplished through live bacteria expressing $7 a$-dehydroxylase or through direct enteral administration of secondary bile acids. The latter approach has proved successful in curing a single case of recurrent ileal pouchitis due to refractory CDI with ursodeoxycholic acid.[31]

\section{Competition for Resources}

As competition for similar ecological niches is believed to be an important mechanism of effective probiotics, strains that compete with toxigenic $C$. difficile represent a promising therapeutic avenue. In particular, non-toxogenic $C$. difficile (NTCD), which presumably shares the closest nutritional requirements, has shown efficacy as a method of CDI prevention.

A phase 2 randomized, double-blind, placebo-controlled study was conducted on NTCD-M3 spores for the prevention of first recurrence of CDI [32] Patients were randomized to a 14day course of three different doses of NTCD-M3 spores or placebo. Of 168 patients, CDI recurrence was $30 \%$ in the placebo group vs $11 \%$ in the combined NTCD-M3 groups (odds ratio $0.28 ; 95 \%$ CI $0.11-0.69 ; P=.006)$. CDI recurrence was lower in those who developed colonization with NTCD (31\% vs. $2 \%)$. NTCD colonization declined substantially after completion of therapy, so CDI protection may be transient without prolonged or repeated courses. There is also a concern that NTCD may acquire toxin production capabilities through horizontal gene transfer, as has been shown in vitro.[33]

\section{Refinements to Fecal Microbiota Transplantation: use of fecal derivatives for the treatment of CDI}

Treatment with undefined consortia of fecal bacteria that are quality-controlled and semistandardized is another avenue of therapy being explored. RBX2660 is a standardized stoolderived microbial suspension containing live bacteria in a cryopreservative derived from screened healthy donors. This microbial suspension can be stored frozen and then thawed and delivered to patients via retention enema.

A phase 2 single-arm study of 34 patients assessed safety and efficacy of RBX2660 in nonimmunocompromised patients with $\searrow$ wo recurrences of CDI.[34] Patients with persistent diarrhea after a first RBX2660 dose could receive a second dose within ten days. Of 31 patients evaluated for efficacy, 14 (45\%) required a second treatment, and 27 of 31 (87.1\%) had treatment success after one or two doses. This study shows the promise of a 
standardized fecal microbial suspension that is easy to administer, though the patient sample was small and the first dose efficacy was suboptimal. However, a follow-up phase $2 b$ trial of 150 subjects receiving two doses of RBX2660 versus placebo failed to meet its primary end point of absence of CDI at 56 days (61\% vs $45.5 \%, P=0.152)$.[35] Larger randomized trials will be needed to further assess efficacy for recurrent CDI.

Other strategies are being developed to repopulate the gut microbiota by methods other than the administration of viable microbes. SER-109 consists of purified spores, generally from bacteria of the Firmicutes phylum, collected from the stool of healthy, pre-screened donors formulated as a capsule. A single-arm, open-label phase 1b study of SER-109 was performed in 30 patients with $\geq$ three prior episodes of CDI. [36] Therapy was well tolerated, and 26 of 30 patients met the primary endpoint of no CDI recurrence within eight weeks. Analysis of microbiota composition by $16 \mathrm{~S}$ rRNA sequencing revealed rapid alterations, with expansion of Firmicutes and amplification of organisms not contained in SER-109, such as Bacteroidetes.

However, as SER-109 is still derived from human stool, it remains relatively undefined with inherent variability in the microbial composition from donor to donor. The efficacy of SER-109 failed to validate in the yet-to-be published phase 2 study's results.[37] The company is now conducting a randomized, double-blind, placebo-controlled, parallel group study of SER-109 as well as a phase 1b study on SER-262, a defined microbial preparation with 12 different types of bacteria in spore form, for prevention of recurrent CDI.[38, 39]

Another approach to administer bacteriotherapy without the transfer of viable microorganisms is through the use of microbe-free fecal filtrates. In the study by Ott et al, fecal supernatant was passed through multiple sequential filters, with a final pore size of 0.2 $\mu \mathrm{m}$.[40] No bacterial growth was detected after cultivation of the resulting product. All five patients treated for recurrent CDI experienced rapid clinical cure with a single administration and remained symptom-free for the six-month follow-up period. 16s rRNA sequencing revealed rapid and complex shifts in gut microbial composition. Proteome analysis of the fecal filtrate demonstrated a wide array of predominantly human-derived proteins and bacteriophages. Combined with a trial that did not demonstrate FMT's efficacy over standard therapy for acutely recurrent CDI, this study underscores the importance of further research into the relative importance of the various stool components in CDI treatment.[41, 42]

\section{Bacteriophage Therapy}

Bacteriophage therapy is another intriguing avenue for effective treatment of multidrug resistant bacteria. It is a particularly attractive option for CDI because of its targeted mechanism of action and lack of significant impact on the microbiota. Research has been hampered by a lack of identified lytic phages specific for $C$. difficile and concerns of using temperate phages that could potentially integrate viral nucleic acid into host DNA. A study by Nale et al investigated the ability of seven different phages, alone and in combination, to reduce the bacterial load of 80 different strains of $C$. difficile representing 21 different ribotypes.[43] The optimal phage combination was strain specific, but multiple three and four phage combinations were able to completely lyse in vitro $C$. difficile cultures within 2- 
5 hours. Mice treated with combination phage therapy survived longer and had a four-log reduction in colonic $C$. difficile bacterial and spore counts.

Major challenges with phage therapy include designing a phage that lacks integrase activity, to reduce risk of transmission of mobile genetic elements (i.e. drug resistance genes), and demonstrating human safety and efficacy.

\section{Conclusion}

Prior clinical trials involving traditional single and small combination probiotic agents have shown modest success in risk reduction of CDI in high-risk patients receiving systemic antibiotics. However, clinicians have been slow to adopt them into practice due to conflicting individual study results and a wide array of heterogeneous products. While incorporating them into treatment is appropriate based on the current body of data and has already been trialed by some hospitals, the search remains for an agent that is easy to administer and provides consistent, durable protection against CDI that is replicated in multiple clinical trials and real-world studies.[44]

The widespread availability of genomic and metabolomic analysis and recent developments in computational modeling result in a more precise understanding of the effects of targeted introduction of probiotics.[30, 45, 46] This will allow for more rational design of agents that specifically manipulate the microbiota to ameliorate dysbiotic changes. Since the native microbiome extends beyond bacteria and fungi and healthy stool includes many nonmicrobial components, further exploration of alternative treatments involving phage therapy and purified microbiota-derived small molecules is also warranted. An evolving understanding of the dynamics of the complex microbial community will allow for creation of better products to promote and restore homeostasis of the intestinal microbiota in the setting of antibiotic stress.

\section{Acknowledgements}

Funding

KR and VBY are supported by grants from the National Institute of Allergy and Infectious Diseases at the National Institutes of Health [grant numbers R21- AI120599 and U01-AI124255].

\section{References}

1. Lessa FC, et al., Burden of Clostridium difficile infection in the United States. N Engl J Med, 2015 372(9): p. 825-34. [PubMed: 25714160]

2. Zimlichman E, et al., Health care-associated infections: a meta-analysis of costs and financial impact on the US health care system. JAMA Intern Med, 2013 173(22): p. 2039-46. [PubMed: 23999949]

3. Biggest Threats Antibiotic/Antimicrobial Resistance. CDC. 2017 Accessed 10 Sept 2017.

4. Loo VG, et al., Host and pathogen factors for Clostridium difficile infection and colonization. N Engl J Med, 2011 365(18): p. 1693-703. [PubMed: 22047560]

5. Alasmari F, et al., Prevalence and risk factors for asymptomatic Clostridium difficile carriage. Clin Infect Dis, 2014 59(2): p. 216-22. [PubMed: 24755858]

6. Vonberg RP, et al., Infection control measures to limit the spread of Clostridium difficile. Clin Microbiol Infect, 200814 Suppl 5: p. 2-20. 
7. Theriot CM and Young VB, Interactions Between the Gastrointestinal Microbiome and Clostridium difficile. Annu Rev Microbiol, 2015 69: p. 445-61. [PubMed: 26488281]

8. Abt MC, McKenney PT, and Pamer EG, Clostridium difficile colitis: pathogenesis and host defence. Nat Rev Microbiol, 2016 14(10): p. 609-20. [PubMed: 27573580]

9. Bagdasarian N, Rao K, and Malani PN, Diagnosis and treatment of Clostridium difficile in adults: a systematic review. Jama, 2015 313(4): p. 398-408. [PubMed: 25626036]

10. Abou Chakra CN, et al., Risk factors for recurrence, complications and mortality in Clostridium difficile infection: a systematic review. PLoS One, 2014 9(6): p. e98400. [PubMed: 24897375]

11. McFarland LV, From yaks to yogurt: the history, development, and current use of probiotics. Clin Infect Dis, 201560 Suppl 2: p. S85-90. [PubMed: 25922406]

12. Hill C, et al., Expert consensus document. The International Scientific Association for Probiotics and Prebiotics consensus statement on the scope and appropriate use of the term probiotic. Nat Rev Gastroenterol Hepatol, 2014 11(8): p. 506-14. [PubMed: 24912386]

13. Hudson LE, et al., Gleaning Insights from Fecal Microbiota Transplantation and Probiotic Studies for the Rational Design of Combination Microbial Therapies. Clin Microbiol Rev, 2017 30(1): p. 191-231. [PubMed: 27856521]

14. Shen NT, et al., Timely Use of Probiotics in Hospitalized Adults Prevents Clostridium difficile Infection: A Systematic Review With Meta-Regression Analysis. Gastroenterology, 2017 152(8): p. 1889-1900.e9.

** Meta-analysis of 19 clinical trials using PRISMA guidelines found a 50\% decreased risk of CDI amoung those treated with probiotics without significant heterogeneity or publication bias. Risk reduction was even greater for the subgroup who began probiotics within 1-2 days of antibiotics (RR 0.32 , 95\% CIs $0.22-0.48$ ), making a compelling case for more widespread probiotic adoption in high-risk populations.

[PubMed: 28192108]

15. Cohen SH, et al., Clinical practice guidelines for Clostridium difficile infection in adults: 2010 update by the society for healthcare epidemiology of America (SHEA) and the infectious diseases society of America (IDSA). Infect Control Hosp Epidemiol, 2010 31(5): p. 431-55. [PubMed: 20307191]

16. Surawicz CM, et al., Guidelines for diagnosis, treatment, and prevention of Clostridium difficile infections. Am J Gastroenterol, 2013 108(4): p. 478-98; quiz 499. [PubMed: 23439232]

17. Rao K and Safdar N, Fecal microbiota transplantation for the treatment of Clostridium difficile infection. J Hosp Med, 2016 11(1): p. 56-61. [PubMed: 26344412]

18. Rao K and Young VB, Fecal microbiota transplantation for the management of Clostridium difficile infection. Infect Dis Clin North Am, 2015 29(1): p. 109-22. [PubMed: 25677705]

19. Ehrhardt S, et al., Saccharomyces boulardii to Prevent Antibiotic-Associated Diarrhea: A Randomized, Double-Masked, Placebo-Controlled Trial. Open Forum Infectious Diseases, 2016 3(1): p. ofw011.

20. Barker AK, et al., A randomized controlled trial of probiotics for Clostridium difficile infection in adults (PICO). J Antimicrob Chemother, 2017.

21. Gibson GR, et al., Dietary modulation of the human colonic microbiota: updating the concept of prebiotics. Nutr Res Rev, 2004 17(2): p. 259-75. [PubMed: 19079930]

22. Slavin J, Fiber and prebiotics: mechanisms and health benefits. Nutrients, 2013 5(4): p. 1417-35. [PubMed: 23609775]

23. Ratsep M, et al., A combination of the probiotic and prebiotic product can prevent the germination of Clostridium difficile spores and infection. Anaerobe, 2017 47: p. 94-103. [PubMed: 28465256]

24. Valdes-Varela L, et al., Effect of Bifidobacterium upon Clostridium difficile Growth and Toxicity When Co-cultured in Different Prebiotic Substrates. Frontiers in Microbiology, 2016 7: p. 738. [PubMed: 27242753]

25. Aktories K, Schwan C, and Jank T, Clostridium difficile Toxin Biology. Annu Rev Microbiol, 2017 71: p. 281-307. [PubMed: 28657883] 
26. Ripert G, et al., Secreted Compounds of the Probiotic Bacillus clausii Strain O/C Inhibit the Cytotoxic Effects Induced by Clostridium difficile and Bacillus cereus Toxins. Antimicrobial Agents \& Chemotherapy, 2016 60(6): p. 3445-54.

* An M-protease with C. difficile inhibitory properties was isolated from Bacillus clausii Strain O/C, making it a promising candidate for future probiotic trials.

[PubMed: 27001810]

27. Spinler JK, et al., Next-generation probiotics targeting Clostridium difficile through precursordirected antimicrobial biosynthesis. Infect Immun, 2017.

28. Hanchi H, et al., Inhibition of MRSA and of Clostridium difficile by durancin 61A: synergy with bacteriocins and antibiotics. Future Microbiol, 2017 12: p. 205-212. [PubMed: 28262046]

29. Winston JA and Theriot CM, Impact of microbial derived secondary bile acids on colonization resistance against Clostridium difficile in the gastrointestinal tract. Anaerobe, 2016 41: p. 44-50. [PubMed: 27163871]

30. Buffie CG, et al., Precision microbiome reconstitution restores bile acid mediated resistance to Clostridium difficile. Nature, 2015 517(7533): p. 205-8. [PubMed: 25337874]

31. Weingarden AR, et al., Ursodeoxycholic Acid Inhibits Clostridium difficile Spore Germination and Vegetative Growth, and Prevents the Recurrence of Ileal Pouchitis Associated With the Infection. J Clin Gastroenterol, 2016 50(8): p. 624-30. [PubMed: 26485102]

32. Gerding DN, et al., Administration of spores of nontoxigenic Clostridium difficile strain M3 for prevention of recurrent C. difficile infection: a randomized clinical trial. Jama, 2015 313(17): p. 1719-27. [PubMed: 25942722]

33. Brouwer MS, et al., Horizontal gene transfer converts non-toxigenic Clostridium difficile strains into toxin producers. Nat Commun, 2013 4: p. 2601. [PubMed: 24131955]

34. Orenstein R, et al., Safety and Durability of RBX2660 (Microbiota Suspension) for Recurrent Clostridium difficile Infection: Results of the PUNCH CD Study. Clin Infect Dis, 2016 62(5): p. 596-602.

* RBX2660, a quality-controlled stool-based product resulted in $87 \%$ treatment success in a small phase 2 trial. It offers some advantages over traditional FMT, such as standardized production through good manufacturing practice and ease of administration through enema.

[PubMed: 26565008]

35. Dubberke Er, L.C.O.R.K.S.H.G.F.J. Efficacy and Safety of RBX2660 for the Prevention of Recurrent. in IDWeek 2016. 2016 Idsa.

36. Khanna S, et al., A Novel Microbiome Therapeutic Increases Gut Microbial Diversity and Prevents Recurrent Clostridium difficile Infection. Journal of Infectious Diseases, 2016 214(2): p. 173-81.

** SER-109, or purified Firmicutes spores, showed promise in the prevention of recurrent CDI in a phase $1 \mathrm{~b}$ trial, with data suggesting expansion of traditionally commensal microbiota and reduction in potentially pathogenic Enterobacteriaceae. Larger trials will be needed to establish efficacy.

[PubMed: 26908752]

37. Press Releases Investor \& Media. Seres Therapeutics 2017; Available from: http:// ir.serestherapeutics.com/phoenix.zhtml?c=254006\&p=irol-newsArticle \&ID=2240833. Accessed 10 Sept 2017.

38. SER-262 Versus Placebo in Adults With Primary Clostridium Difficile Infection to Prevent Recurrence - Full Text View - ClinicalTrials.gov. 201710 Sept 2017]; Available from: https:// clinicaltrials.gov/ct2/show/NCT02830542.

39. ECOSPOR III - SER-109 Versus Placebo in the Treatment of Adults With Recurrent Clostridium Difficile Infection - Full Text View - ClinicalTrials.gov 2017 Accessed 13 Oct 2017.

40. Ott SJ, et al., Efficacy of Sterile Fecal Filtrate Transfer for Treating Patients With Clostridium difficile Infection. Gastroenterology, 2017 152(4): p. 799-811.e7. 
** Proof of concept that highly filtered stool, devoid of any vegetative bacteria, is capable of durable CDI cure in a small cohort of patients, including several immunocompromised hosts. Sterile fecal filtrate removes risk of pathogen transmission and suggests that specific host or bacterial small molecules play an important role in $\mathrm{C}$. difficile colonization resistance.

[PubMed: 27866880]

41. Kelly CR, et al., Effect of Fecal Microbiota Transplantation on Recurrence in Multiply Recurrent Clostridium difficile Infection: A Randomized Trial. Ann Intern Med, 2016 165(9): p. 609-616. [PubMed: 27547925]

42. Hota SS, et al., Oral Vancomycin Followed by Fecal Transplantation Versus Tapering Oral Vancomycin Treatment for Recurrent Clostridium difficile Infection: An Open-Label, Randomized Controlled Trial. Clin Infect Dis, 2017 64(3): p. 265-271. [PubMed: 28011612]

43. Nale JY, et al., Bacteriophage Combinations Significantly Reduce Clostridium difficile Growth In Vitro and Proliferation In Vivo. Antimicrob Agents Chemother, 2016 60(2): p. 968-81. [PubMed: 26643348]

44. Maziade PJ, Pereira P, and Goldstein EJ, A Decade of Experience in Primary Prevention of Clostridium difficile Infection at a Community Hospital Using the Probiotic Combination Lactobacillus acidophilus CL1285, Lactobacillus casei LBC80R, and Lactobacillus rhamnosus CLR2 (Bio-K+). Clin Infect Dis, 201560 Suppl 2: p. S144-7. [PubMed: 25922400]

45. Jenior ML, et al., Clostridium difficile Colonizes Alternative Nutrient Niches during Infection across Distinct Murine Gut Microbiomes. mSystems, 2017 2(4).

46. Bucci V, et al., MDSINE: Microbial Dynamical Systems INference Engine for microbiome timeseries analyses. Genome Biology, 2016 17(1): p. 121. [PubMed: 27259475]

47. Carasi P, et al., Surface proteins from Lactobacillus kefir antagonize in vitro cytotoxic effect of Clostridium difficile toxins. Anaerobe, 2012 18(1): p. 135-42. [PubMed: 22126976]

48. Castagliuolo I, et al., Saccharomyces boulardii protease inhibits the effects of Clostridium difficile toxins A and B in human colonic mucosa. Infect Immun, 1999 67(1): p. 302-7. [PubMed: 9864230]

49. Solomon K, The host immune response to Clostridium difficile infection. Ther Adv Infect Dis, 2013 1(1): p. 19-35. [PubMed: 25165542]

50. Goldenberg JZ, et al., Probiotics for the prevention of Clostridium difficile-associated diarrhea in adults and children. Cochrane Database Syst Rev, 2013(5): p. Cd006095. [PubMed: 23728658]

51. Dubberke ER, et al., Clearance of Vancomycin-Resistant Enterococcus Concomitant With Administration of a Microbiota-Based Drug Targeted at Recurrent Clostridium difficile Infection. Open Forum Infectious Diseases, 2016 3(3): p. ofw133. [PubMed: 27703995] 


\section{Key points}

- $\quad$ Probiotic use may reduce incident CDI in high risk populations by as much as $50 \%$, though prior clinical trials have yielded conflicting results

- $\quad$ There are several promising new approaches to prevention of CDI including combining probiotics with prebiotics, use of strains that directly inhibit $C$. difficile, modulation of bile acid metabolism to improve colonization resistance, niche competition, and bacteriophage therapy.

- $\quad$ Refinements to FMT, including standardized stool-derived products, purified spores, and sterile fecal filtrate, blur the line between probiotics and FMT.

- Important safety and efficacy concerns still impede the widespread deployment and acceptance of probiotics. 


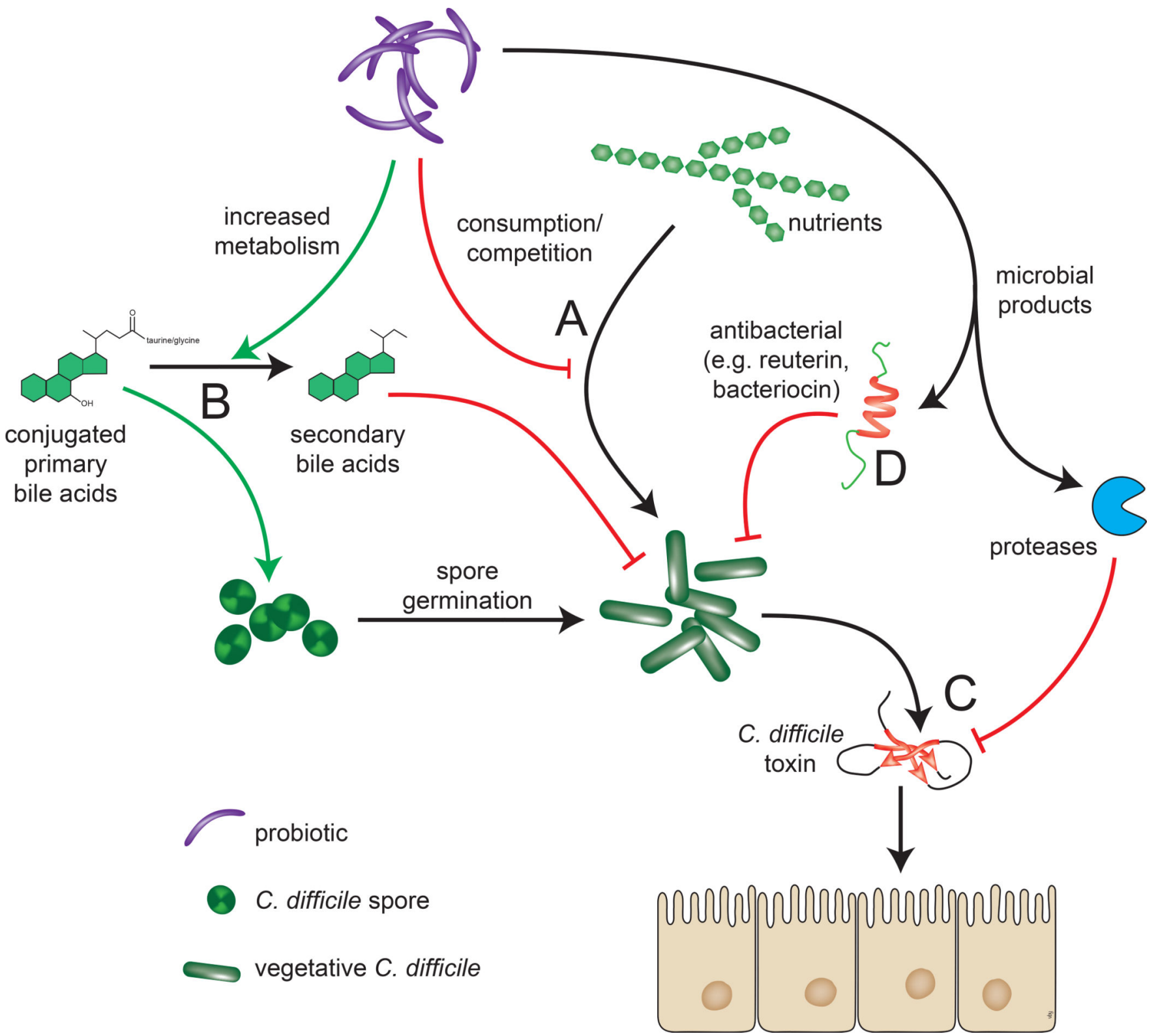

FIGURE 1: Mechanisms of probiotic protection against CDI.

There are multiple potential mechanisms by which probiotics could modulate the onset and course $C$. difficile infection. A) Competition for resources. For example, increased levels of nitrogen-containing amino acids, sialic acid, succinate, and host-derived glycans, and decreases in short chain fatty acids provide a favorable environment for growth of vegetative C. difficile and their consumption by probiotics could be inhibitory. B) Decreased metabolism of primary to secondary bile acids by $7 a$-dehydroxylase promotes germination of $C$. difficile spores, and this can be counteracted by administering bacteria that have such activity. C) The $C$. difficile toxins TcdA and TcdB are responsible for the symptoms of disease and these can be counteracted through secretion of inhibitory compounds. D) Bacteroicins, a class of antimicrobial peptides, can be directly secreted by probiotics. Non- 
specific effects of probiotics that alter $\mathrm{pH}$, increase mucosal IgA levels, or increase mucin production can also inhibit $C$. difficile (not depicted).[47-49] 
TABLE 1:

Microorganism or microorganism-derived therapies that are actively in use or development for prevention of CDI.

\begin{tabular}{|l|l|l|l|}
\hline & Advantages & Disadvantages & References \\
\hline $\begin{array}{l}\text { Single or small combination of } \\
\text { bacterial/fungal strains }\end{array}$ & $\begin{array}{l}\text { Extensive clinical trial and real-world } \\
\text { experience Safety well-established }\end{array}$ & $\begin{array}{l}\text { Modest efficacy Lack of standardization, } \\
\text { heterogeneity among products }\end{array}$ & {$[11,14,50]$} \\
\hline $\begin{array}{l}\text { Non-toxogenic } C \text {. difficile } \\
\text { (NTCD) }\end{array}$ & $\begin{array}{l}\text { Safe and effective in phase II study Provides } \\
\text { nutritional niche competition }\end{array}$ & $\begin{array}{l}\text { Transient NTCD colonization provides } \\
\text { short-lived protection }\end{array}$ & {$[32]$} \\
\hline Fecal consortia (e.g. RBX2660) & $\begin{array}{l}\text { Deliver complex native microbial } \\
\text { communities (mimicking FMT) May } \\
\text { promote clearance of other MDROs }\end{array}$ & $\begin{array}{l}\text { Undefined, variable product Theoretical } \\
\text { risk of transmitted infection }\end{array}$ & {$[34,51]$} \\
\hline Firmicute spores (e.g. SER-109) & Very low risk of transmitted infection & $\begin{array}{l}\text { Undefined, variable product Limited } \\
\text { clinical trial data }\end{array}$ & {$[36]$} \\
\hline Sterile Fecal Filtrate & $\begin{array}{l}\text { Very low risk of transmitted infection } \\
\text { Unaffected by systemic antibiotics } \\
\text { supportive data }\end{array}$ & {$[40]$} \\
\hline Bacteriophages & $\begin{array}{l}\text { Unaffected by systemic antibiotics Narrow } \\
\text { spectrum of activity }\end{array}$ & $\begin{array}{l}\text { Lack of supportive human data Risk of } \\
\text { unanticipated gene exchange }\end{array}$ & {$[43]$} \\
\hline
\end{tabular}

